\title{
Formigas em Grafo: Um Jogo Educacional para Apoio ao Ensino e Aprendizagem dos Algoritmos de Busca em Largura e Busca em Profundidade
}

\author{
Adriel Vieira Santos' ${ }^{1}$, Andressa Bezerra Ferreira ${ }^{1}$ \\ ${ }^{1}$ Instituto Federal de Educação, Ciência e Tecnologia do Ceará \\ Fortaleza-CE, Brasil \\ \{adriel.kltegmail.com, andressa.ferreiradifce.edu.br\}
}

\begin{abstract}
In Computer Science, when it is necessary to consider a set of objects and the relationships between pairs of these objects, it is possible to use a data structure known as a graph. Because it is an abstract structure, learning graph theory and understanding its algorithms is complex. Therefore, this work aims to develop, evaluate and present an educational game called Formigas em Grafo. The aim of the game is to facilitate the understanding of the behavior of search algorithms in depth and width, through practice in a concrete environment. The game developed was evaluated according to the PAJED (Program for the Evaluation of Educational Digital Games) and obtained results that indicate a high learning potential.
\end{abstract}

Resumo. Na Ciência da Computação, quando é necessário considerar um conjunto de objetos e as relações entre pares desses objetos é possivel utilizar uma estrutura de dados conhecida como grafo. Por se tratar de uma estrutura abstrata, a aprendizagem da teoria de grafos e o entendimento de seus algoritmos é algo complexo. Diante disso, este trabalho tem como objetivo desenvolver, avaliar e apresentar um jogo educacional chamado Formigas em Grafo. $O$ intuito do jogo é facilitar o entendimento do comportamento dos algoritmos de busca em profundidade e largura, por meio da prática em um ambiente concreto. O jogo desenvolvido foi avaliado segundo o PAJED (Programa de Avaliação de Jogos Digitais Educacionais) e obteve resultados que indicam um alto potencial de aprendizado.

\section{Contextualização}

Uma das principais estruturas de dados existentes na Ciência da Computação é o grafo (Ziviani, 2004). Um grafo $G$ pode ser definido como um par $G=(V, A)$, em que $V$ é um conjunto de vértices e A é um conjunto de arestas. Por se tratar de uma estrutura abstrata, a aprendizagem da teoria dos grafos e o entendimento de seus algoritmos (e.g., algoritmo de busca em largura, algoritmo de busca em profundidade) não é trivial. 
Devido a importância da área, muitos trabalhos foram desenvolvidos e, de acordo com Oliveira (2019), é possível notar o surgimento de um consenso na comunidade acadêmica de que o uso de ferramentas visuais pode facilitar o ensino e aprendizagem de grafos.

Com base nesse cenário e considerando a importância do estudo da teoria dos grafos, este trabalho tem como objetivo desenvolver, avaliar e apresentar um jogo educacional chamado Formigas em Grafo. O jogo tem o intuito de facilitar o entendimento do comportamento dos algoritmos de busca em profundidade e busca em largura, por meio da prática em um ambiente concreto.

Pretende-se avaliar o jogo e sua aplicabilidade por meio do PAJED (Programa de Avaliação de Jogos Digitais Educacionais), proposto por Santos e Alves (2019).

\section{Fundamentação Teórica}

De acordo com Cormen (2002), os grafos são um tipo de estrutura combinatória com diversas aplicações no mundo real. Dentre elas, destacam-se, principalmente, os problemas de locomoção (e.g., determinar o menor caminho entre dois pontos).

Segundo Ziviani (2004), um grafo $\mathrm{G}=(\mathrm{V}, \mathrm{A})$ pode ser classificado como direcionado ou não direcionado. Quando o grafo é dito direcionado, o conjunto A é uma relação binária em V. Já quando o grafo é não direcionado, o conjunto A é formado por pares de vértices não ordenados. Isso significa que se o par $(\mathrm{u}, \mathrm{v})$ pertence a $\mathrm{A}$, o par $(\mathrm{v}, \mathrm{u})$ também pertence a $\mathrm{A}$. Nos grafos direcionados (Figura 1: Grafo B) os

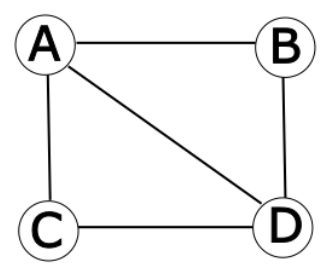

Grafo A

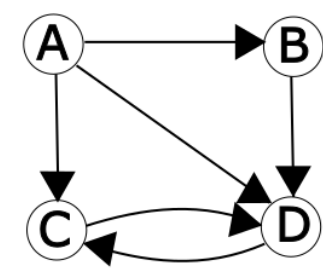

Grafo B arcos possuem uma seta indicando o relacionamento, já no não direcionado

(Figura 1: Grafo A), os arcos não possuem setas.

Figura 1: Grafo não direcionado e direcionado

Dentre os principais algoritmos associados ao estudo dos grafos estão o algoritmo de busca em largura e o algoritmo de busca em profundidade. Segundo Ziviani (2004), a busca em profundidade é uma forma de visitação que utiliza a estratégia de buscar sempre o mais fundo no grafo. Considerando um vértice inicial qualquer, a busca explora todos os vértices mais recentementes descobertos de forma recursiva até não existir vértice algum, e então volta para trás, visitando os vértices restantes fazendo uso da mesma estratégia.

Ainda de acordo com Ziviani (2004), dado um vértice inicial, o algoritmo de busca em largura visita de forma sistemática todos os vértices adjacentes ao inicial antes de avançar para vértices mais distantes. Ainda segundo o autor, esse método de busca é bastante importante, pois é a base para o algoritmo de Dijkstra e o algoritmo de Prim, ambos essenciais para a teoria dos grafos.

\section{Trabalhos Correlatos}


No trabalho de Figueiredo e Figueiredo (2011), é proposto um jogo chamado WarGrafos. O jogo é baseado no jogo WAR e funciona da seguinte forma: cada equipe recebe um objetivo relacionado a conceitos da teoria de grafos e precisa alcançá-lo durante uma partida. Esses objetivos são relacionados a conceitos como grafos completos, bipartidos, planares, grau de um vértice entre vários outros. Ganha quem consegue alcançar o objetivo primeiro. Para validar a ferramenta os autores realizaram um teste com 49 alunos de uma turma, sendo 22 repetentes. Quando a ferramenta foi aplicada a quantidade de reprovação foi reduzida para 9 alunos, desconsiderando os desistentes.

Em Oliveira (2018), é proposto um jogo educacional chamado Puzzle Grafo. O jogo foi desenvolvido para a plataforma Android e é baseado em um jogo de labirinto. O objetivo é executar o algoritmo de busca em profundidade no labirinto movendo uma bolinha pelos vértices de um grafo antes que o tempo acabe. O modelo de avaliação usado no trabalho foi MEEGA+ que avalia a qualidade do jogo educacional por meio de perguntas subjetivas com foco na experiência do jogador e na percepção da aprendizagem. Dentre os pontos contabilizados, a satisfação dos usuários obteve $94 \%$ de aceitação e os objetivos de aprendizagem 97\%, sendo esses os maiores valores contabilizados dentre os critério abordados.

Em Barbosa, De Freitas Nunes e Gondim (2014), são propostos dois jogos, DEG4TREE ABB e DEG4TREE AVL. O primeiro jogo aborda a inserção e a remoção de nós em uma árvore binária de busca com o intuito de emergir um submarino inflando boias com ar no caso da inserção, e trocando essas boias quando elas furam, aplicando o algoritmo de remoção. $\mathrm{O}$ segundo jogo trata das árvores AVL e consiste em um restaurante, onde o discente deve manter os copos equilibrados utilizando os métodos de balanceamento. Para realizar a avaliação foi utilizado o modelo de aceitação de tecnologia TAM (Technology Acceptance Model) que avalia a utilidade percebida e facilidade de uso percebida. Após a avaliação foram obtidos média 6.26 com desvio padrão de 0.32 para utilidade percebida e média 6.48 com desvio padrão de 0.31 para facilidade de uso percebida. De acordo com o autor, a maior média alcançável por meio da fórmula usada é 7. Portanto, quanto mais próximo a avaliação está do número 7 , melhor o resultado da avaliação.

Dentre os trabalhos analisados, é possível perceber que o único que aborda temática semelhante ao deste é Oliveira (2018). No entanto, o autor foca apenas na busca em profundidade, desconsiderando os aspectos de busca em largura. A ferramenta apresentada por Figueiredo e Figueiredo (2011), por sua vez, se distingue das demais, pois se concentra nos aspectos teóricos relacionados às propriedades dos grafos. Os autores não utilizaram nenhum método existente na literatura para realizar a avaliação, fazendo apenas duas perguntas para avaliar o jogo.

Já o trabalho realizado por Barbosa, De Freitas Nunes e Gondim (2014), desdobra-se sobre uma subcategoria de grafos acíclicos, chamados de árvores binárias, e embora não trate de algoritmos de busca como largura e profundidade, o trabalho apresenta evidências e características para a abordagem do problema de ensino e aprendizagem de algoritmos e estrutura de dados por meio de jogos digitais educacionais. 
Mediante esse contexto, percebe-se a oportunidade e relevância de propor um jogo educacional que aborda não apenas a busca em profundidade, mas também a busca em largura da teoria dos grafos. Além disso, torna-se imprescindível apresentar uma avaliação metódica do jogo proposto.

\section{O Jogo}

O jogo Formigas em Grafo $^{1}$ é constituído de um mapa repleto de formigueiros e possui como objetivo realizar a alimentação desses vários formigueiros antes que eles entrem em colapso. No jogo, cada formigueiro possui um tempo de vida que é reduzido dependendo da sua localização no mapa.

No mapa, por sua vez, cada formigueiro representa um vértice do grafo. Para interligar os formigueiros é utilizado o feromônio da formiga, que conecta os pontos e serve para indicar o caminho que o personagem deve percorrer para chegar ao formigueiro desejado. Portanto, o feromônio representa as arestas que conectam os vértices em um grafo.

Em cada vértice existe também uma barra de energia. A barra é utilizada para indicar o tempo de vida de cada formigueiro. Logo, a alimentação de cada formigueiro (i.e., vértice), deve ser feita antes que o nível da barra chegue ao final.

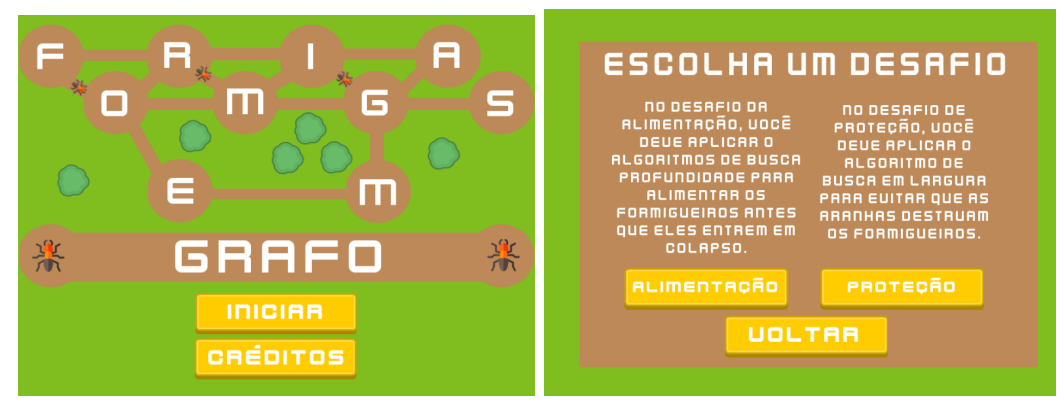

Figura 2: O jogo Formigas em Grafo

Atualmente estão implementadas doze fases de jogo, sendo seis para o método de busca em largura e seis para busca em profundidade. Para cada fase existe um mapa diferente e o jogador é livre para se mover por ele, mas ao sair da trilha entre os formigueiros, a formiga que o representa fica lenta e desorientada, comprometendo o seu deslocamento e dificultando a chegada até os formigueiros. Além disso a agilidade do jogador é posta a prova, devido as aranhas que tentam impedir que a formiga cumpra seu objetivo.

Para resolver cada fase, o jogador deve visitar os formigueiros seguindo a mesma ordem que o algoritmo seguiria se fosse executado sobre um grafo. Quando o jogador não segue o algoritmo, os formigueiros acabam entrando em colapso antes do previsto, fazendo com que o jogador não consiga vencer a fase, sendo necessário portanto, realizar o

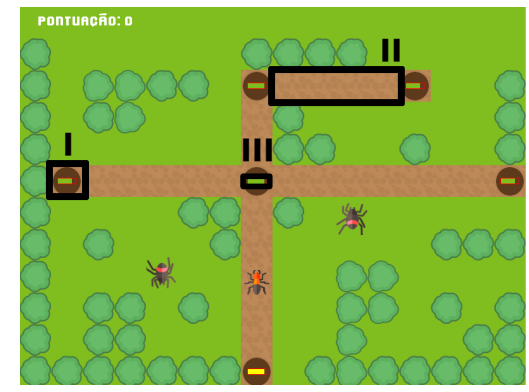

Figura 3: Vértices e arestas processo de visitação do início. O jogador sempre perde se pelo menos um dos formigueiros

\footnotetext{
${ }^{1}$ Repositório público do jogo educacional Formigas em Grafo: https://github.com/omitidoParaFinsDeRevisao
} 
entrar em colapso. Na Figura 3 é possível notar os formigueiros (Vértices), o feromônio (arestas) e o tempo de vida de cada formigueiro, representados por I, II e III respectivamente.

No grupo de fases referentes ao algoritmo de busca em profundidade, todos os grafos representados nos mapas são acíclicos. Optou-se por grafos com essa característica, pois durante os desafios, as informações sobre os estados dos vértices do grafo ficam implícitos. Uma vez que o jogo tem como foco demonstrar e possibilitar o treino do comportamento do algoritmo, o discente não se prejudica pela não apresentação de detalhes (e.g., tempo de descoberta, tempo de término, estado de visitação do vértice e lista de antecessores de um vértice).

Ao iniciar uma fase de jogo relativa ao algoritmo de busca em profundidade, o jogador percebe que os formigueiros que estão nos extremos do mapa consomem a barra de energia com mais rapidez, sendo necessário, alimentá-los primeiro. Quando o formigueiro do extremo de um determinado mapa de busca em profundidade é alimentado, o formigueiro adjacente anterior ao já alimentando se torna um novo extremo, fazendo com que a sua barra de energia diminua em maior velocidade. Desse modo, mantém-se a característica da busca em profundidade, segundo a teoria dos grafos.

Já nas fases que abordam a busca em largura, existem grafos cíclicos e acíclicos. Diferentemente da busca em profundidade, inicialmente, todos os formigueiros possuem a mesma necessidade, fazendo com que a barra de energia de cada formigueiro seja consumida na mesma velocidade. Neste modo do jogo, o jogador inicia em uma posição qualquer do mapa. Após a alimentação do primeiro formigueiro, é iniciado uma reação em cadeia que desperta a necessidade dos outros formigueiros adjacentes aquele alimentado. $\mathrm{O}$ efeito da reação mantém a propriedade da busca em largura, pois acelera o consumo da barra de energia de todos os adjacentes ao formigueiro já alimentado.

Como o algoritmo da busca em largura utiliza uma fila de forma explícita, após a alimentação de um formigueiro qualquer, todos os formigueiros adjacentes ao alimentado, sofrem uma alteração na cor da barra de energia, passando de verde para amarelo, com o objetivo de lembrar ao jogador, qual o grupo de formigueiros ele deve alimentar.

Considera-se importante mencionar que, antes de iniciar qualquer uma das fases, o jogador visualiza uma breve descrição sobre os algoritmos e uma pequena animação da mecânica do jogo. O intuito dessas informações iniciais é orientar o usuário sobre as regras que ele precisa seguir.

\section{Avaliação do Jogo}

Para alcançar o objetivo proposto neste trabalho, foram realizadas duas importantes atividades: o desenvolvimento do jogo educacional Formigas em Grafo e a avaliação do mesmo utilizando o Programa de Avaliação de Jogos Digitais Educacionais (PAJED), disponível em Santos (2019).

Segundo Santos (2019), o PAJED tem como objetivo ser uma ferramenta para avaliação de jogos educacionais que, a partir de um questionário, define o potencial de aprendizado de um jogo, indicando se o mesmo é útil ou não para o ensino e aprendizado. A ferramenta aborda oito dimensões consideradas, de acordo com a literatura, importantes para 
avaliação de jogos educacionais. São elas: narrativa, desafios em níveis, feedback imediato, objetivos educacionais, níveis de interatividade, integração de conceitos, curva de aprendizagem e práticas colaborativas.

Para cada dimensão os autores da ferramenta atribuíram um peso. Ao final da avaliação é possível calcular o que os autores do método chamam de potencial de aprendizado.

$$
\begin{gathered}
P A=(11.29 * F E+15.30 * O A+9.92 * N A+12.97 * N I+14.67 * I C+14.76 * C A+8.11 * P C+ \\
12.97 * N D) / 10 .
\end{gathered}
$$

Onde: $\mathrm{FE}=$ Nota obtida sobre o Feedback Imediato; $\mathrm{OA}=$ Nota obtida sobre os Objetivos de Aprendizagem; NA $=$ Nota obtida sobre a Narrativa; NI = Nota obtida sobre o Nível de Interatividade; IC = Nota obtida sobre a Integração de Conceitos; CA $=$ Nota obtida sobre a Curva de Aprendizagem; $\mathrm{PC}=$ Nota obtida sobre a Prática Colaborativa; $\mathrm{ND}=$ Nota obtida sobre os Níveis de Desafios. Utilizando a Escala Likert, as notas do PAJED são atribuídas conforme apresentado nas Tabelas 1 e 2.

Tabela 1 - Notas PAJED pela Escala Likert
\begin{tabular}{|c|c|}
\hline Item & Nota \\
\hline Concordo Totalmente & 10,0 \\
\hline $\begin{array}{c}\text { Concordo Parcialmente } \\
\text { Não Concordo, nem } \\
\text { Discordo }\end{array}$ & 7,5 \\
\hline Discordo Parcialmente & 5,0 \\
\hline Discordo Totalmente & 0,0 \\
\hline
\end{tabular}

Tabela 2 - Escala do Potencial de Aprendizado segundo PAJED
\begin{tabular}{|c|c|}
\hline Item & Nota \\
\hline $80 \leq \mathrm{PA} \leq 100$ & Potencial de Aprendizagem Muito Alto \\
\hline $60 \leq \mathrm{PA}<80$ & Potencial de Aprendizagem Alto \\
\hline $40 \leq \mathrm{PA}<60$ & Potencial de Aprendizagem Moderado \\
\hline $20 \leq \mathrm{PA}<40$ & Potencial de Aprendizagem Baixo \\
\hline $0 \leq \mathrm{PA}<20$ & Potencial de Aprendizagem Muito Baixo \\
\hline
\end{tabular}

Os alunos que participaram da avaliação são discentes do curso de Bacharelado em Sistemas de Informação do Omitido para fins de revisão. O curso não dispõe de uma disciplina específica de grafos, mas o conteúdo faz parte da ementa da disciplina de Algoritmos e Estruturas de Dados, ofertada para discentes do terceiro semestre.

As aulas são ministradas de maneira expositiva por meio de recursos visuais, onde o professor costuma solicitar a implementação dos seguintes algoritmos: busca em largura, busca em profundidade, algoritmo de Dijkstra, algoritmo de Prim e Algoritmo de Kruskal. Os alunos são incentivados a implementarem os métodos em tecnologias diversas e ao final das implementações o resultado é apresentado em sala por meio de seminários.

A avaliação do jogo foi realizada em sala de aula com a participação de 11 (onze) discentes. Antes que os alunos utilizassem o jogo e realizassem a avaliação, o conteúdo sobre os algoritmos de busca em largura e em profundidade foi abordado com intuito de apresentar o seu funcionamento e possíveis aplicações. Em seguida, o jogo foi apresentado para todos e, ao final, foi utilizado um formulário do Google para coletar as avaliações anonimamente de acordo com o método apresentado. Optou-se pelo anonimato para que os estudantes se sentissem mais à vontade durante o processo de avaliação. 


\section{Análise dos Resultados}

Para cada discente que participou do experimento foi contabilizado um potencial de aprendizado e, em seguida, a média do potencial de aprendizado calculada foi arredondada para o menor inteiro mais próximo. Com isso, o potencial de aprendizagem obtido foi em média 92 pontos. Diante disso, acordo com o PAJED, o jogo educacional Formigas em Grafo possui um potencial muito alto de aprendizado.

Na Figura 4 é possível visualizar a distribuição das avaliações para cada dimensão do método. Os gráficos em pizzas são organizados da esquerda para direita, iniciando na dimensão 1 (um) e terminando na dimensão 8 (oito).

Apesar do resultado animador (i.e., 92 pontos PAJED), com base nos gráficos é possível notar que as dimensões 1, 4 e 7 receberam algumas avaliações baixas, sendo elas: "Não Concordo, Nem Discordo" e "Discordo Parcialmente". Na dimensão 1, a avaliação recebeu um "Discordo Parcialmente", possivelmente porque o feedback ocorre de maneira implícita, uma vez que, quando o jogador erra, os formigueiros que mantém as características das buscas são penalizados de forma que o jogador não consiga vencer.

Já na dimensão 4, a mesma avaliação se repete e, possivelmente, decorre do fato da dificuldade razoavelmente alta de todo o jogo. Para torná-lo atrativo, todas as fases possuem um nível de dificuldade alto e crescente. Na maioria dos casos, o jogador vence nos últimos segundos.

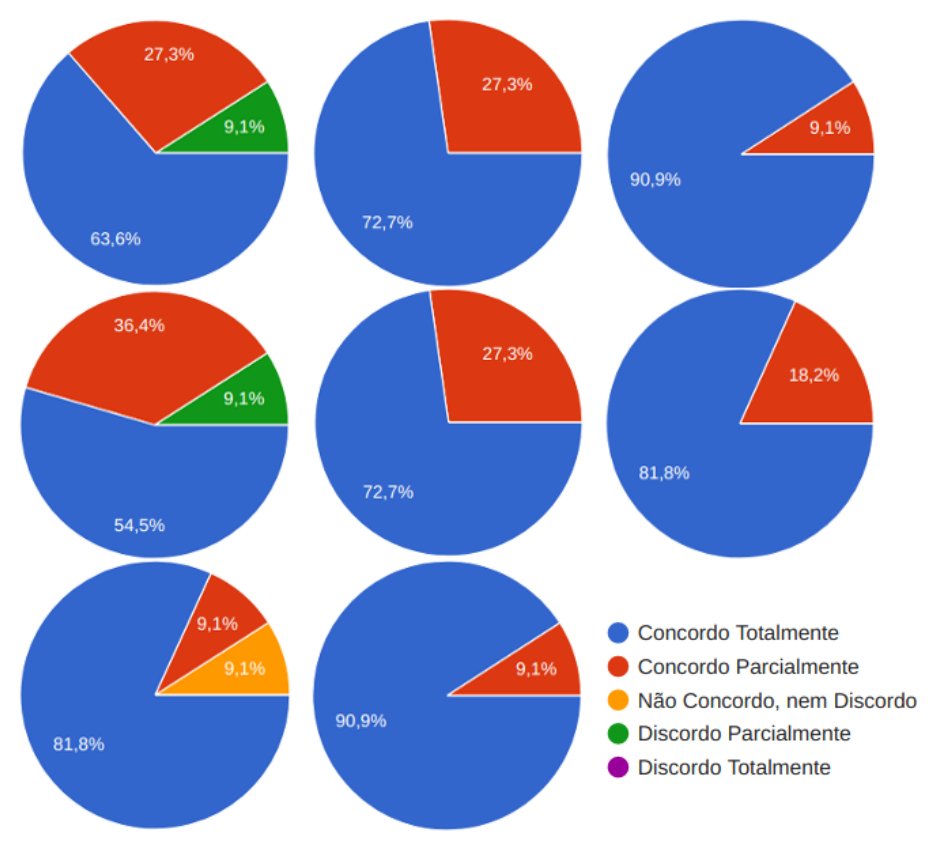

Figura 4: Distribuição das respostas para cada dimensão do PAJED

A última dimensão recebeu a avaliação mais baixa: "Não Concordo, Nem Discordo". O motivo da avaliação é bastante compreensivo, pois o jogo é para um único jogador. Entretanto, como os desafios necessitam de estratégias para serem resolvidos, isso pode vir a favorecer a troca de ideias entre os discentes.

\section{Considerações Finais}

Segundo Cormen (2002), algoritmos e estruturas de dados são temáticas de extrema importância para a Ciência da Computação. De acordo com Ziviani (2004), uma das estruturas de dados mais relevantes é o grafo. Na literatura, é possível identificar diversos 
estudos que abordam a necessidade do uso de recursos visuais para o ensino e aprendizagem da teoria dos grafos [(Barboza, 2013), (Oliveira, 2019), (Figueiredo e Figueiredo (2011)].

Diante desse contexto, este trabalho teve como objetivo desenvolver, avaliar e apresentar um jogo educacional para auxiliar o ensino e a aprendizagem dos algoritmos de busca em largura e busca em profundidade, ambos presentes na teoria dos grafos. A avaliação foi realizada por meio do método PAJED, apresentado em Santos e Alves (2019), e obteve o potencial de aprendizagem alto. O jogo está disponível em um repositório público e pode ser baixado de forma gratuita.

Apesar dos indícios positivos, uma série de trabalhos futuros se faz necessária. Dentre eles: 1) a ampliação do conteúdo informativo sobre os algoritmos, de modo a incluir pseudocódigos, análise de complexidade e aplicações, 2) o aprimoramento dos gráficos do jogo, almejando uma melhor atratividade visual e 3) a análise da ferramenta como um recurso alternativo para ser aplicado em metodologias de aprendizagem híbrida, especificamente no modelo caracterizado por sala de aula invertida apresentada em Valente (2014).

\section{Referências}

BARBOSA, Weider Alves; JÚNIOR, Paulo Afonso Parreira. Um mapeamento sistemático sobre ferramentas de apoio ao ensino de algoritmo e estruturas de dados. In: Brazilian Symposium on Computers in Education (Simpósio Brasileiro de Informática na Educação-SBIE). 2013. p. 406.

BRYFCZYNSKI, Sam et al. Teaching data structures with BeSocratic. In: Proceedings of the 18th ACM conference on Innovation and technology in computer science education. ACM, 2013. p. 105-110.

CORMEN, Thomas H. et al. Algoritmos: teoria e prática. Editora Campus, v. 2, p. 2, 2002.

FIGUEIREDO, Roberto Tenorio; FIGUEIREDO, C. Wargrafos - jogo para auxílio na aprendizagem da disciplina de teoria dos grafos. X Simpósio Brasileiro de Games e Entretenimento Digital (SBGames 2011), 2011.

OLIVEIRA, Álvaro Alves de. Puzzle grafo: um jogo educacional mobile para auxiliar ao aprendizado do algoritmo de busca em profundidade. 2018. 23 f. TCC (Graduação) Sistemas de Informação Instituto Federal de Educação, Ciência e Tecnologia do Ceará/ Campus Crato, Crato, 2018. Disponível em: <biblioteca.ifce.edu.br/index.asp?codigo_sophia=84007>. Acesso em: 17 out. 2019.

SANTOS, Willian; ALVES, Lynn Rosalina Gama. PAJED: Um Programa de Avaliação de Jogos Digitais Educacionais. Anais do Seminário de Jogos Eletrônicos, Educação e Comunicação, v. 3, n. $1,2019$.

SANTOS, Rodrigo P. et al. O uso de ambientes gráficos para ensino e aprendizagem de estruturas de dados e de algoritmos em grafos. In: Anais do XVI Workshop sobre Educação em Computação, XXVIII Congresso da Sociedade Brasileira de Computação. sn, 2008. p. 157-166.

VALENTE, José Armando. Blended learning e as mudanças no ensino superior: a proposta da sala de aula invertida. Educar em Revista, n. 4, p. 79-97, 2014. 